\title{
Improved Methods of Signal Processing Used in Low-Coherent Systems
}

\author{
M. JEDRZEJEWSKA-SzCZERSKA* \\ Department of Optoelectronics and Electronics Systems \\ Gdańsk University of Technology \\ Narutowicza 11/12, 80-952 Gdańsk, Poland
}

\begin{abstract}
In this article the author presents improved techniques of signal processing used in low-coherent measurement systems. The author proposes to utilize the synthesized sources in the system with the temporal signal processing. It will be shown that the exploit of such a source can substantially reduce the signal-to-noise ratio required to identify the central fringe position. In case of using spectral signal processing, the author proposes to control the position of maximum in the spectral pattern which modifies the phase difference between interfering beams. The results of experimental works will show that described techniques can be an effective method for improving the signal processing in low-coherent measurement systems.
\end{abstract}

PACS numbers: 42.79.--e, 42.81.Pa

\section{Introduction}

In low-coherent measurement systems it is possible to use two kinds of optical signal processing: temporal and spectral. When the temporal signal processing is used, the most critical problem is to identify the position of the central fringe in the interference fringe pattern. It is so important because this position corresponds to the zero of optical path difference, and therefore gives information about the measurand. The measurement signal of low-coherent systems with temporal signal processing is described by equation [1]:

$$
I(\sigma)=I_{0}(\sigma)[1+V(\sigma) \cos (\sigma)],
$$

where $I$ - intensity of the source, $V$ - visibility of the interference fringes, $\delta$ optical path difference.

The measurement signal of such a system using the source with Gaussian power density spectrum is shown in Fig. 1a. It can be seen in Fig. 1a that the intensity difference between the central $\left(I_{00}\right)$ fringe and the first side fringe $\left(I_{01}\right)$

*e-mail: mjedrzej@eti.pg.gda.pl 


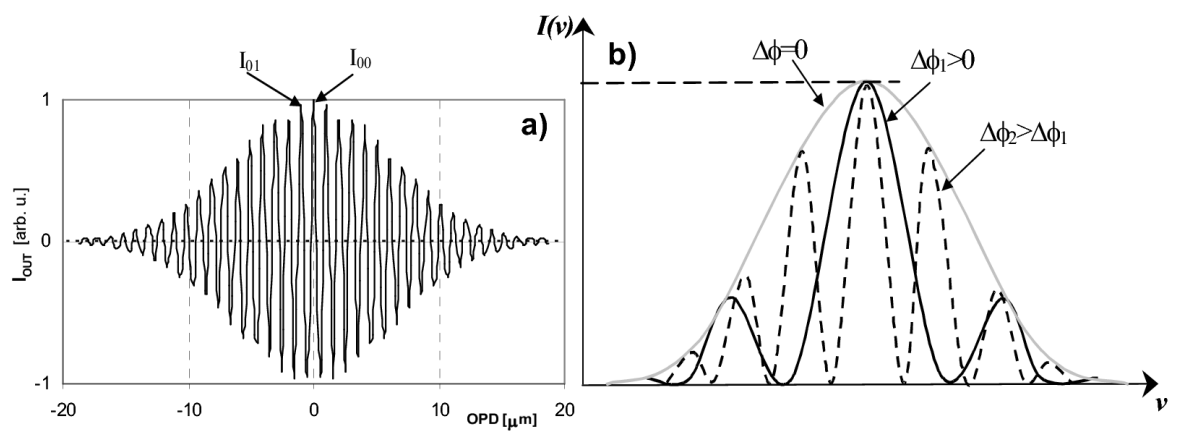

Fig. 1. (a) The measurement signal of a low-coherent system with signal processing in time domain, (b) the measurement signal of low-coherent system with signal processing in spectral domain.

in the fringe packet can be so small that the signal-to-noise ratio required by the system for direct identification of the central fringe should be at the range of $50 \div 60 \mathrm{~dB}[2]$ which is really hard to reach in real optoelectronic systems.

When the spectral signal processing is used, the measurement signal can be described as [3]:

$$
I_{\text {out }}(\nu)=S(\nu)\left[1+V_{0}(\Delta \phi) \cos (\Delta \phi)\right],
$$

where $S(\nu)$ - the spectral distribution of the light source, $V_{0}$ - visibility of interference fringes, $\Delta \phi$ - the phase difference between interfering beams.

If the light source exhibits a Gaussian spectrum, the normalized spectra pattern is predicted to be a cosine function modified by the Gaussian visibility profile, as shown in Fig. 1b.

In the spectral domain signal processing the modulation frequency of the measurement signal gives information about measurand (Eq. (2)), as shown in Fig. 1b [4]. Therefore, it is necessary to use special measurement equipment and mathematical treatment of the measurement signal, which makes this method comparatively expensive, complicated and time-consuming.

\section{Improved method of signal processing}

To overcome the problem connected with signal processing the author proposes the improved methods of signal processing which have been used in the realized low-coherent measurement systems for temperature measuring.

\subsection{Low-coherent measurement system employing the improved method of signal processing in time domain}

In a low-coherent system with time domain signal processing the author utilized the synthesized source which was combined by summation of the autocorrelation function of two superluminescent diodes with parameters: the central wavelengths $\lambda_{1}=1290 \mathrm{~nm}, \lambda_{2}=1560 \mathrm{~nm}$ and the spectral bandwidths 
$\Delta \lambda_{1}=50 \mathrm{~nm}, \Delta \lambda_{2}=45 \mathrm{~nm}$. Those parameters were chosen by the use of a computer simulation which was capable of calculating the optimal parameters of broadband emitters $[5,6]$.

It can be seen in Fig. 2 that identification of the central fringe position becomes considerably easier when synthesized source is used rather than one emitter with a single wavelength, especially when the two sources have the optimum wavelength combination. On the other hand, the low-coherent measurement system with synthesized source (made by summation of autocorrelation function of two sources) is relatively easy to build, compared to other approaches. The utilization of such a source improves all metrological parameters of the system. It can be noted by analyzing the data included in Table.

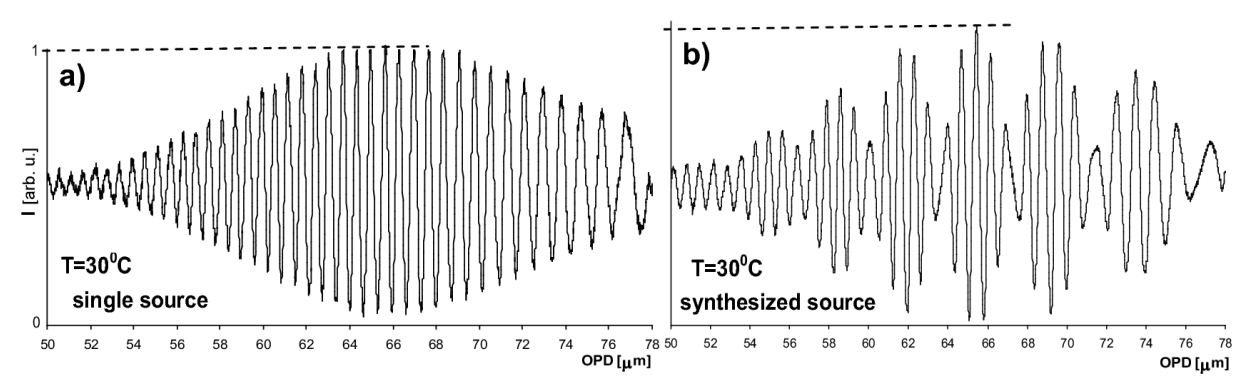

Fig. 2. (a) Measured interference fringes with the use of a single source, (b) measured interference fringes with the use of a synthesized source.

TABLE

Parameters of the low-coherent systems for temperature measuring.

\begin{tabular}{c|c|c}
\hline \hline Parameters & System with a single source & System with a synthesized source \\
\hline $\mathrm{SNR}[\mathrm{dB}]$ & 40 & 19 \\
$S\left[\mu \mathrm{m} /{ }^{\circ} \mathrm{C}\right]$ & 0.228 & 0.310 \\
$B_{y}[\%]$ & \pm 14 & \pm 2.5
\end{tabular}

The exploitation of the synthesized source leads to meaningful decrease in the value of signal-to-noise ratio required for precise, direct identification of the central fringe position in the fringe pattern (SNR). Additionally, the use of such a source results in increasing the sensitivity $(S)$ and decreasing the relative measurement error $\left(B_{y}\right)$ of the measurement process.

\subsection{Low-coherent measurement system employing improved method of signal processing in spectral domain}

Any change of the phase difference between interfering beams reflected from the sensor head depends on temperature changes and occurs in measurement signal [7]. In the spectral domain signal processing the modulation frequency of the 
measurement signal gives information about the measurand. However, the author perceived that any changes of the phase difference between the interfering beams modify not only the modulation frequency of the measurement signal but the position of maxima in the spectral pattern as well, which can be observed in Fig. 3a [5]. The dependence of the spectra pattern of the presented sensor on the temperature over the range from 30 to $40^{\circ} \mathrm{C}$ is plotted in Fig. 3b. The maximum position shifts to the range of the wavelength of higher value during heating. The dependence of the maximum position in the spectral pattern on temperature during cooling and heating is shown in Fig. 3b. It should be noticed that the maximum position change per temperature unit is almost constant over the whole investigated range from 30 to $400^{\circ} \mathrm{C}$ and linear. The correlation coefficient of relationship between the maximum position shift and temperature has a value $R^{2}=0.993$. The described sensor has a sensitivity at the range $S=1.03$ and respond time $t_{1}=2.6$ when the temperature changes from 400 to $25^{\circ} \mathrm{C}$. Hence, the realized temperature sensor confirms its ability for temperature measurement.
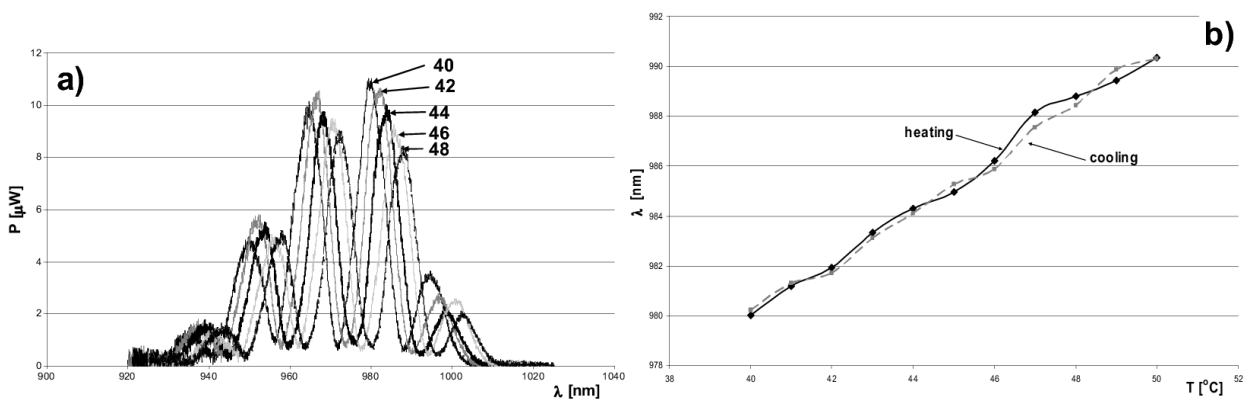

Fig. 3. (a) Measured spectra pattern changes with the change of temperature, (b) measured temperature influence on the maximum position in spectra pattern.

The results of experimental works have shown that the method based on the control of the shift of maximum position in the spectra pattern is useful, especially when the measured optical path difference is too small to modify the frequency of measurement signal modulation [8].

\section{Conclusions}

The subject of this article is improved methods of signal processing used in low-coherent, interferometric measurement systems of physical quantities. In this work, the author presents the analysis of low-coherent measurement systems with the signal processing in the phase and spectral domain. The use of low-coherent sources synthesized from individual broadband emitters has been proposed, to improve accuracy with which the central fringe of the interferogram is determined. Systems measuring temperature employing phase and spectral domain signal processing are presented as well as the results of experimental verification of their 
performance. Using experimental studies, the author has demonstrated that substantial improvements in metrological parameters of discussed measurement systems can be attained by employing the source synthesized by the summation of autocorrelation function of two sources. In case of using spectral signal processing, the author proposes to control the position of maximum in the spectral pattern which modifies the phase difference between interfering beams.

The experimental works have shown that the described techniques can be an effective method for improving the signal processing in low-coherent measurement systems.

\section{Acknowledgments}

The current investigation is a part of a research project No. 3 T11B 08429 supported by the Ministry of Science and Higher Education.

\section{References}

[1] K.T.V. Grattan, B.T. Meggit, Optical Fiber Sensor Technology, Kluwer Academic Publ., Boston 2000.

[2] J. Rao, Opt. Lett. 18, 462 (1993).

[3] S. Egerov, A. Mamaev, I. Likhachiev, Proc. SPIE 2594, 193 (1996).

[4] P. Hlubina, P. Stejskal, Proc. SPIE 4517, 267 (2001).

[5] M. Jedrzejewska-Szczerska, B.B. Kosmowski, R. Hypszer, J. Phys. IV (France) 137, 107 (2006).

[6] S. Chen, K. Grattan, B. Meggit, A. Palmer, Electron Lett. 29, 334 (1993).

[7] D.V. Bogomolov, V. Voloschiniv, K.B. Yushkon, Mol. Quant. Acoust. 28, 299 (2007).

[8] M. Jedrzejewska-Szczerska, R. Bogdanowicz, M. Gnyba, R. Hypszer, B.B. Kosmowski, Europ. Phys. J. Special Topics 154, 107 (2008). 